\title{
Analytical Technique in the Boundary Element Method for 3D Self-Consistent Problems of Electron Optics
}

\author{
Valentin Ivanov
}

Institute of Computational Technologies SB RAS, Novosibirsk, Russia

The comparative analysis of modern mathematical models for $3 \mathrm{D}$ problems in electron optics is presented. The new approach to solve the electron optics problems in three dimensions is presented. It is based on the principal ray method suggested by G. Grinberg in 1948. That perspective approach was not realized before for full three-dimensional electron optic systems, probably because of the complexity of its mathematical apparatus. We describe the analytical technique of the boundary element method (BEM) for the field evaluation, and 3-rd order aberration expansion for the trajectory analysis. The first version of such computer code "OPTICS-3", and some results of numerical simulations with this code were presented.

\section{Comparative analysis of different numerical models}

The problem formulations in electron optics are very multifarious. It is well common to split them onto two main classes: nonlinear self-consistent problems of high-current beams, and precision problems of the image electronic devices. First class includes high-powered devices for the energy transformation, but second one includes the image transformation instruments. Different physical problems generate the set of different mathematical models because of different requirements for the numerical solution. Thus, the efficiency of any particular model depends on the features of the physical problem formulation. In the paper we provide the comparative analysis of computational efficiency for four mathematical models: pseudo viscosity method, "pipe-current" method, aberration approach and principal ray method.

The pseudo viscosity method is the sort of relaxation methods. It is based on use the non-stationary algorithm to find the stationary solution as the limit of non-stationary physical process. Normally it is formulated as the combination of Maxwell's solver for the field problems, and "particle-in-cell" (PIC) model for the particle dynamics. This method is extremely useful to detect the instability regimes for the almost stationary problems, but it is very inefficient for the true stable problems comparing with the other methods mentioned above.

The "pipe-current" method (PCM) [1] is the most popular method to simulate the stationary optics of high-current relativistic beams. It includes the set of equations for self-consistent problem: field equation (Poisson's equation), motion equation (Lorentz's equation), and continuity equation for the charge and current conservation law. This method has slightly different formulations for laminar and non-laminar flows of charged particles.

The aberration approach (AA) is a version of more general perturbation theory applying to the light and charge particle optics. The general idea of this method is based on the expansion of the solution for trajectories on the set of small parameters of the problem, which gives the main advantages of high accuracy and reduces the computation time on many orders comparing the "pipe-current" method. Main disadvantage of this approach is that it is applicable for the narrow enough beams only. 
The principal ray method (PRM) [2] removes the paraxial limitation of the aberration approach, as it combines all positive features of the "pipe-current" and of aberration methods in terms of the accuracy and efficiency for the solution. It is applicable as for stationary as for non stationary problems, selfconsistent and weak- current image optics also.

The computational efficiency for different methods can be expressed in terms of CPU-time $\mathbf{T}$ need to solve some typical problem. This time consists of two parts: time of computation for the field evaluation $\mathbf{T}_{\text {field }}$, and time for the particles tracking $\mathbf{T}_{\text {part }}$. First item depends on the numerical method. Let $\mathbf{N}_{\mathbf{s p}}$ is a total number of nodes for spatial mesh, and $\mathbf{N}_{\mathbf{s f}}$ is a number of nodes for the boundary surface in discretization of the problem. Then $\mathbf{T}_{\text {field }} \sim \mathbf{N}_{\mathrm{sp}} \ln \mathbf{N}_{\mathrm{sp}}$ for the Finite Difference Method (FDM) and for the Finite Element Method (FEM). The difference is FDM uses the structured mesh, but FEM uses non structured mesh, and so the value $\mathbf{N}_{\mathbf{s p}}$ is much less for second case. Boundary Element Method (BEM) uses spatial mesh covers whole volume of the problem, but the beam volume only. This case the value $\mathbf{N}_{\text {sp }}$ is much less than for FDM and FEM. In case of FEM the value $\mathbf{T}_{\text {field }} \sim \mathbf{N}_{\text {sf }}$ for weak-current problem, and $\mathbf{T}_{\text {field }} \sim \mathbf{N}_{\mathrm{sp}}+\mathbf{N}_{\text {sf }}$ per one iteration over space charge for high-current problem. All methods should multiply the value by $\mathbf{N}_{\text {iter }}$ for a stationary problem and by $\mathbf{N}_{\text {time }}$ for non-stationary one, where $\mathbf{N}_{\text {iter }}$ is a total number of non linear iterations over space charge, but $\mathbf{N}_{\text {time }}$ is a number of time steps.

The time $\mathbf{T}_{\text {part }} \sim \mathbf{N}_{\text {part }} * \mathbf{N}_{\text {time }}$ for the PIC model, where $\mathbf{N}_{\text {part }}$ is total number of macro particles. In case of the PCM we have $\mathbf{T}_{\text {part }} \sim \mathbf{N}_{\text {traj }} * \mathbf{N}_{\text {iter }}$, where $\mathbf{N}_{\text {traj }}$ is a total number of trajectories represent the beam. Normally the curvilinear surface of emitter is divided onto $\mathbf{N}_{\mathbf{t}}$ by $\mathbf{N}_{\mathbf{s}}$ intervals in $\mathbf{t}-$ and s- spatial directions. We also should make divisions onto $\mathbf{N}_{\boldsymbol{\theta}}$ by $\mathbf{N}_{\boldsymbol{\omega}}$ intervals to take into account the angular distributions for polar $\theta$ and azimuth $\omega$ angles, and onto $\mathbf{N}_{\varepsilon}$ intervals to take into account the energy distribution for emitted particles. Thus, total number of trajectories for PCM model $\mathbf{N}_{\text {traj }}=\mathbf{N}_{\mathbf{t}} * \mathbf{N}_{\mathbf{s}} * \mathbf{N}_{\boldsymbol{\theta}} *$ $\mathbf{N}_{\boldsymbol{\omega}} * \mathbf{N}_{\varepsilon}$. The AA model uses the polynomial expansion onto small parameters - initial angles and energy. This case the angular and energy distributions are taking into account automatically. The motion equation is the second-order differential equation, so $\mathbf{N}_{\text {traj }}=\mathbf{4}$ for $3 \mathrm{D}$ case always. In contrast to PCM this case we should integrate numerically as 4 paraxial trajectories as a few coefficients of expansion. Total number of those coefficients depends on the order of expansion, and on the number of small parameters. The advantage of AA-model comparing the PCM is it can be used as for stationary as for non stationary problem (so called "tau-variation method" TVM [3]). Thus, the AA is the fastest algorithm. Its efficiency can be 3-4 orders higher comparing with PIC and PCM models. Another advantage is the accuracy of AA simulations should be 3-4 orders higher because of analytical representation of aberration expansions onto initial small parameters of emission or injection of particles. The PRM is just a modification of AA model, when the whole emitter surface is divided onto $\mathrm{N}$ sub domains to keep the accuracy of aberration expansions in case of wide beams. Typical values for $\mathrm{N}$ are $1 \div 7$.

Figure 1 and Figure 2 demonstrate the efficiency of different models for simulation of the same diode klystron gun with parameters I $=263 \mathrm{~A}, \mathrm{U}=500 \mathrm{kV}$. To reach the same accuracy of modeling the PCM model used 5 trajectories, 6 iterations on space charge, but PIC model used 3500 macro particles, and 1200 time steps. The difference in CPU-time is an order of magnitude. 


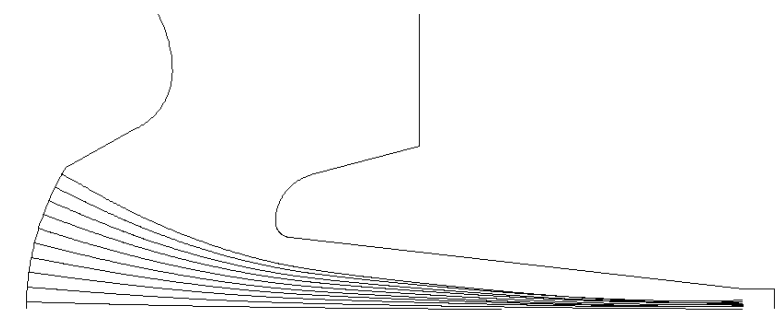

Figure 1. PCM+BEM nodels. $\mathrm{CPU}$-time $=5$ $\min$

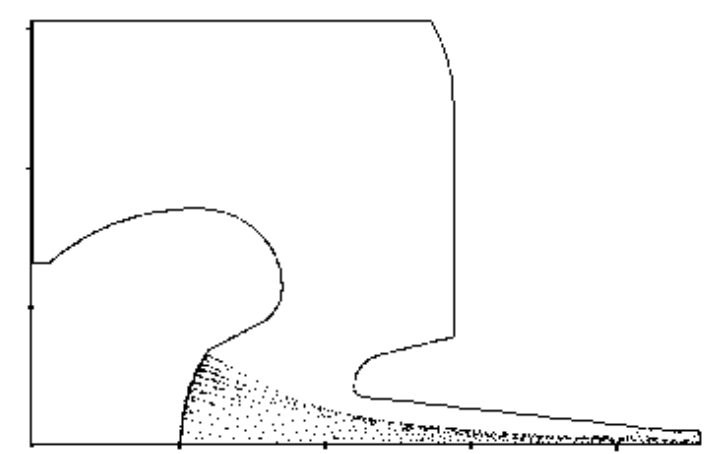

Figure 2. PIC+FDM models. CPU-time $=65$ $\min$.

\section{Principal Ray method}

Suggested numerical model is a composition of BEM for the field evaluation and PRM for the trajectory analysis. The BEM intensive uses the analytical technique [4] to evaluate the integrals over the surface and volume elements formed in discretization of the integral representation for scalar potential $\varphi$ of electric field

$$
\varphi(r)=\frac{1}{4 \pi \varepsilon_{0}}\left(\int_{S} \sigma\left(s^{\prime}\right) G\left(r, s^{\prime}\right) d s^{\prime}+\int_{V} \rho\left(s^{\prime}\right) G\left(r, r^{\prime}\right) d V^{\prime}\right), s^{\prime} \in S, \quad r^{\prime} \in V,
$$

where $r$ - is an observation point, $\varepsilon_{0}$ - is a vacuum permittivity, $\sigma$-is surface charge density, but $\rho$ - is space charge density. The Green's function $G=1 / R$, where $R-$ is a distance between an observation point and source points $s^{\prime}$ and $r^{\prime}$. This numerical model was successive used in simulation of X-band sheet-beam klystron [5].

Integral representation (1) has an important advantage in aberration approach for the electron optic problems. It takes a possibility to evaluate the arbitrary-order derivatives with no accuracy loss as $\mathrm{G}$ is an analytical function.

The principal ray method uses the expansion on time variable to represent the off-axis trajectories. We assume the beam axis is curvilinear one in general case. The motion equation of charged particle with the mass $m$ and charge $e$ in curvilinear coordinate system can be written in the form

$$
\ddot{r}_{\alpha}(\tau)=e / m\left(-\nabla_{\alpha} U(\vec{r}(\tau), \tau)+v_{\beta} B_{\gamma}(\vec{r}(\tau), \tau) g_{\alpha \beta \gamma}\right),
$$

where $r$-is a Cartesian coordinate, $\tau-$ is a time-of-flight, $U-$ is an electrostatic potential, $v-$ is a velocity of particle, $B$ - is a magnetic induction, $g-$ is a metric tensor, and Greek indices $\alpha, \beta, \gamma$ accept the values $x, y, z$.

The $3^{\text {rd }}$ order expansion on an arbitrary small parameter $\mu$ has a form

$$
\begin{aligned}
r_{\alpha}(\tau, \mu) & =r_{\alpha}\left(\tau, \mu_{0}\right)+\left.\frac{\partial r_{\alpha}(\tau, \mu)}{\partial \mu^{i}}\right|_{\mu=\mu_{0}}\left(\mu^{i}-\mu_{0}^{i}\right)+\left.\frac{1}{2} \frac{\partial^{2} r_{\alpha}(\tau, \mu)}{\partial \mu^{i} \partial \mu^{j}}\right|_{\mu=\mu_{0}}\left(\mu^{i}-\mu_{0}^{i}\right)\left(\mu^{j}-\mu_{0}^{j}\right)+ \\
& +\left.\frac{1}{6} \frac{\partial^{3} r_{\alpha}(\tau, \mu)}{\partial \mu^{i} \partial \mu^{j} \partial \mu^{k}}\right|_{\mu=\mu_{0}}\left(\mu^{i}-\mu_{0}^{i}\right)\left(\mu^{j}-\mu_{0}^{j}\right)\left(\mu^{k}-\mu_{0}^{k}\right)+\ldots
\end{aligned}
$$

Here $\mu_{0}$ - is a value of small parameter at the basic trajectory (beam axis) with zero initial velocity, upper indices $i, j, k=1, \ldots, n$, where $n-$ is a total number of small parameters. The partial derivatives are 
the aberration coefficients. The sense of small parameters is determined by the emission or injection initial conditions. They can be the components of off-axis distance, initial angles or energy, and start time of the particles. The equations for the aberration coefficients can be obtained with substitution of the expansion (3) to the motion equation (2). Thus, we should integrate numerically small set of equations: the motion equation and a set of variation equations for the aberration coefficients instead integration of huge number of motion equation for PCM model. This approach can drastically reduce the CPU-time of simulation (5-6 orders of magnitude), and keep the precision accuracy of computations. It was realized in the code "OPTICS-3" [6]. Figure 3 shows the trajectories for the night-vision device consists of the photo cathode, anode, the gap between them, screen, and external magnetic field excited with round solenoid. Next Figure 4 shows some $3^{\text {rd }}$-order aberration coefficients as the functions of $\mathrm{z}-$ coordinate: B - spherical aberration, E - distortion, $\mathrm{P}$ - chromatic, Q - chromatic-position. The CPUtime for that simulation was about 2 minutes.
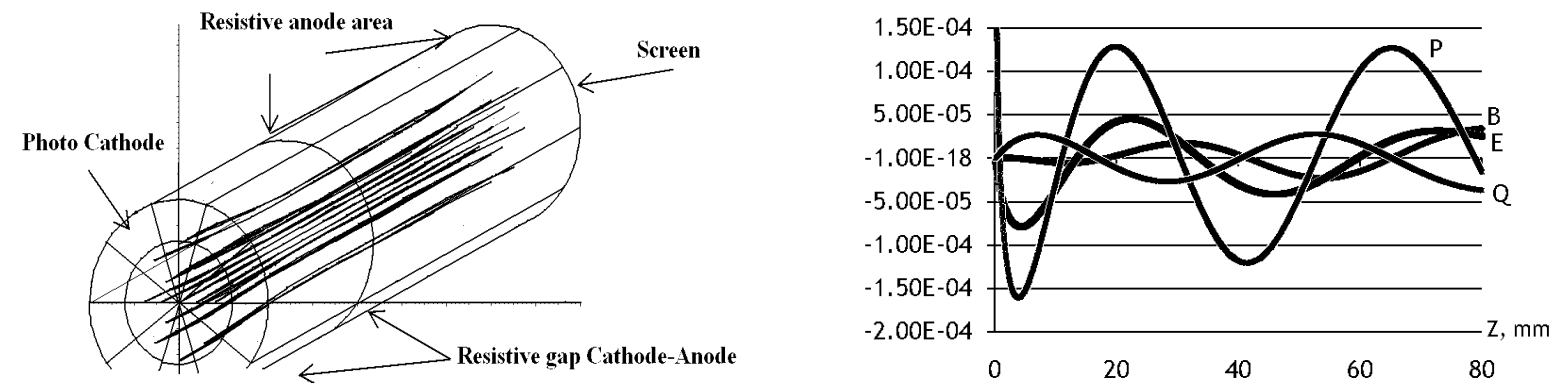

Figure 3. Trajectories in electron optical Figure 4. Some aberration coefficients. converter.

An example of modeling for diode sheet-beam gun of X-band SLAC klystron with using the PCM model $[7,8]$ is presented at Figure 5. CPU-time for that simulation was more than 1 hour. These two examples convincing demonstrate the comparative efficiency of different numerical models.
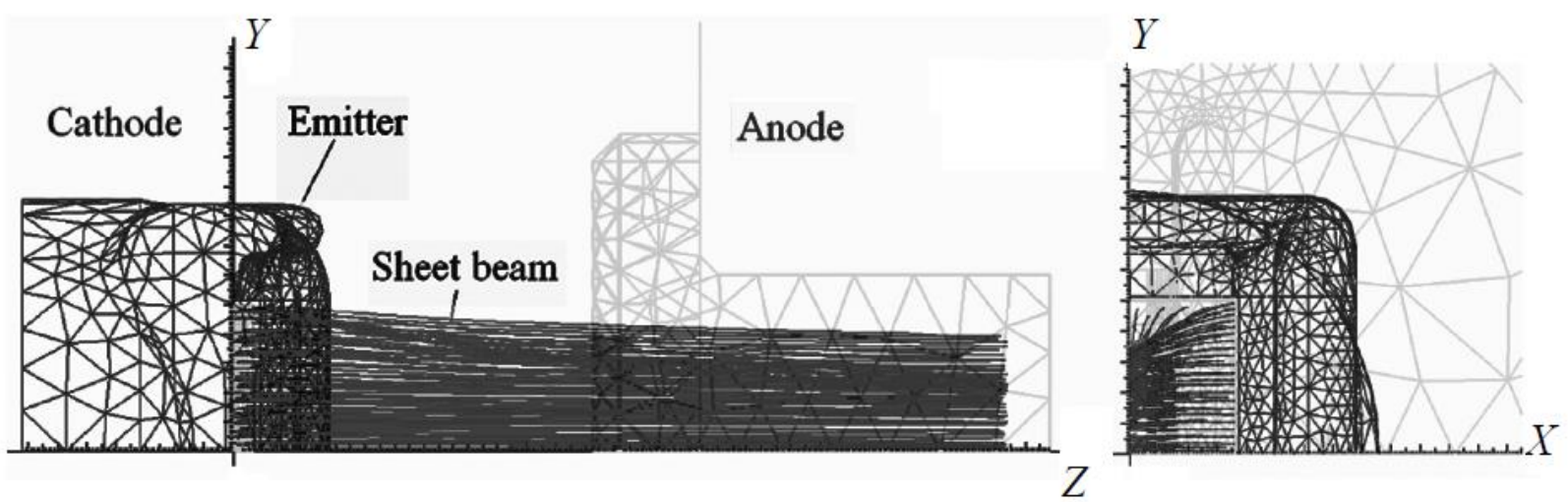

Figure 5. Klystron gun simulation with using BEM for the field evaluation, and PCM for the trajectory analysis.

\section{Analytical evaluation the integrals over the triangle surface elements}

The integrals for electrostatic potential with linear distribution of charge density over the triangle were evaluated by E. Okon and R. Harrington [9] 


$$
\varphi(x, y, z) \equiv \frac{1}{4 \pi \varepsilon} \iint_{\Omega} \frac{\left(s_{1} \tau+s_{2} \eta\right) d \tau d \eta}{\sqrt{\left(x-x^{\prime}(\tau, \eta)\right)^{2}+\left(y-y^{\prime}(\tau, \eta)\right)^{2}+\left(z-z^{\prime}(\tau, \eta)\right)^{2}}} .
$$

The formulas derived by authors are regular. Unfortunately, authors did not provide the expressions for the field gradients. They include the singularities in the vicinity of vertices and edges of triangle. Some of these singularities are artificial ones because they are annulated for pairs of triangles connected smoothly. The others singularities were analyzed and regularized accurately by V. Ivanov [5], [7].

\section{Evaluation the integrals over the volume elements.}

Let us consider the non-uniform parallelepiped mesh in Cartesian coordinates $\left\{x_{i}\right\} \times\left\{y_{j}\right\} \times\left\{z_{k}\right\}$ with linear approximation for the space charge

$$
\begin{aligned}
& \rho(x, y, z)=\left\{\left[\left(\rho_{i+1, j, k}\left(x-x_{i}\right)+\rho_{i, j, k}\left(x_{i+1}-x\right)\right)\left(y_{j+1}-y\right)+\left(\rho_{i+1, j, k}\left(x-x_{i}\right)+\rho_{i, j, k}\left(x_{i+1}-x\right)\right)\right.\right. \\
& \left.\quad\left(y-y_{j}\right)\right]\left(z_{k+1}-z\right)+\left[\left(\rho_{i+1, j, k+1}\left(x-x_{i}\right)+\rho_{i, j, k+1}\left(x_{i+1}-x\right)\right)\left(y_{j+1}-y\right)+\left(\rho_{i+1, j, k+1}\left(x-x_{i}\right)+\right.\right. \\
& \left.\left.\left.\rho_{i, j, k+1}\left(x_{i+1}-x\right)\right)\left(y-y_{j}\right)\right]\left(z-z_{k}\right)\right\} /\left\{\left(x_{i+1}-x_{i}\right)\left(y_{j+1}-y_{j}\right)\left(z_{k+1}-z_{k}\right)\right] .
\end{aligned}
$$

In order to evaluate the potential

$$
\varphi(x, y, z)=\sum_{i, j, k} \int_{x_{i}}^{x_{i+1}} \int_{y_{j}}^{y_{j+1}} \int_{z_{j}}^{z_{k+1}} \rho\left(x^{\prime}, y^{\prime}, z^{\prime}\right) G\left(x, y, z ; x^{\prime}, y^{\prime}, z^{\prime}\right) d x d y d z
$$

we need four moments of space charge

$$
J_{1}=\iiint \frac{x}{R} d x d y d z, J_{2}=\iiint \frac{x y}{R} d x d y d z, J_{3}=\iiint \frac{x y z}{R} d x d y d z, J_{4}=\iiint \frac{1}{R} d x d y d z,
$$

where $R^{2}=\left(x-x^{\prime}\right)^{2}+\left(y-y^{\prime}\right)^{2}+\left(z-z^{\prime}\right)^{2}$. The other integrals are obtained by cyclic change of variables $x, y, z$.

After the change $\tilde{x}=x^{\prime}-x, \tilde{y}=y^{\prime}-y, \tilde{z}=z^{\prime}-z$, and $r^{2}=x^{2}+y^{2}+z^{2}$ all integrals can be evaluated analytically

$$
\begin{aligned}
J_{1}= & \frac{y}{4}\left[r z+\left(x^{2}+y^{2}\right) \ln |z+r|\right]+\frac{x^{2}}{2}\left[z \ln |y+r|+y \ln |z+r|-z+x \operatorname{tg}^{-1}\left(\frac{z}{x}\right)-x \operatorname{tg}^{-1}\left(\frac{z y}{x r}\right)\right] \\
& +\frac{z^{3}}{6} \ln |y+r|+\frac{1}{36}\left[6 x^{2} z-2 z^{3}+6 x^{3}\left(\operatorname{tg}^{-1}\left(\frac{z y}{x r}\right)-\operatorname{tg}^{-1}\left(\frac{z}{x}\right)\right)-3 y\left(y^{2}+3 x^{2} \ln |z+r|\right)\right], \\
J_{2}= & \frac{z r^{3}}{12}+\frac{3}{24}\left(x^{2}+y^{2}\right)\left[z r+\left(x^{2}+y^{2}\right) \ln |z+r|\right], \quad J_{3}=\frac{r^{5}}{15}, \\
J_{4}= & x y \ln |z+r|+y z \ln |x+r|+z x \ln |y+r|-\frac{1}{2}\left[x^{2} \operatorname{tg}^{-1} \frac{y z}{x r}+y^{2} \operatorname{tg}^{-1} \frac{x z}{y r}+z^{2} \operatorname{tg}^{-1} \frac{x y}{z r}\right] .
\end{aligned}
$$

Similar technique was used by V. Ivanov [10] for 2D linear approximation of space charge in Cartesian and cylindrical in 1989. Three-dimensional case for piecewise-constant approximation was studied by J.Quang et al. [9]. 
In order to get the field gradient we should differentiate the integrals (7). By this way. For example, for the component $E_{z}$ we need the following integrals

$$
\begin{aligned}
& J_{1 z}=\iint \frac{d x d y}{r}=-z \operatorname{tg}^{-1} \frac{x y}{z r}+y \ln |x+r|+x \ln |y+r|, \\
& J_{2 z}=\iint \frac{x d x d y}{r}=\frac{1}{2}\left[y r+\left(x^{2}+z^{2}\right) \ln |y+r|\right], J_{3 z}=\iint \frac{z d x d y}{r}=z J_{1 z}, J_{4 z}=\iint \frac{x y d x d y}{r}=\frac{r^{3 / 2}}{3}, \\
& J_{5 z}=\iint \frac{x z d x d y}{r}=z J_{2 z}, J_{6 z}=\iint \frac{x y z d x d y}{r}=\frac{z}{3} r^{3 / 2} .
\end{aligned}
$$

The other integrals are obtained by cyclic change of variables $x, y, z$. We should pay special attention for the singularity problem. Charged cube has singularities in the vicinity of vertices and edges. For linear and higher order approximations the singularities for inner vertices and edges are annulated, but they exist for outer boundary points and edges. Fortunately, the space charge density tends to zero on the beam envelope for high-order approximation, so the singularities are disappear. But singularity problem remains for piece-wise constant approximation, which creates a problem in its use for the electron optic problems. Detail analysis of approximation errors and convergence were done at [4].

In general case the space charge approximation with cubic elements can give significant errors for highcompression beams. Suggested analytical technique can be generalized for general curvilinear mesh fitted with the beam envelope, when we can construct the continuous one-to-one map $T$ for curvilinear coordinates $(u, v, w)$ to Cartesian ones $(x, y, z)$. This case the integration over curvilinear cells will be reduced to evaluation the integrals (6)-(9) with the replacement $\rho(u, v, w) d u d v d w$ to $\rho(x, y, z) J d x d y d z$, where $J-$ is the Jacobean for the coordinate mapping $T$.

\section{References:}

[1] V Ivanov in "Computer Aided Design of Physical Electronic Devices", (Institute of Mathematics of SB RAS Publishing, Novosibirsk).

[2] GA Grinberg in "Selected Questions of Mathematical Theory of electric and magnetic phenomena" (USSR Academy of Science Publishing, Moscow-Leningrad).

[3] M Monastyrsky, Prikladnaya Fizika 3 (1986), p. 7.

[4] V Ivanov, Int. J. Mod. Phys. A 24 (2009), p. 869.

[5] V Ivanov, Nauchnoe priborostroenie 24 (2014), p. 96.

[6] V Ivanov and Y Kulikov in "Topical Problems of Computational Mathematics and computer simulation" (2012) p. 12.

[7] V Ivanov et al, IEEE Particle Accelerator Conference (2001) p. 1213.

[8] V Ivanov et al, IEEE Particle Accelerator Conference (2003) p. 12.

[9] EE Okon and RF Harrington, Int. J. Numer. Meth. Eng. 18 (1982), p. 1821.

[10] V Ivanov in "Proc. Inst. of Mathematics SB RAS vol. 15", (Nauka Publisher, Novosibirsk), (1989) p. 172 .

[11] J Quang et al, Phys. Rev. Spec. Top. Ac. 9 (2006), 44204. 